\title{
Synthesis of Biquadratic Impedances with At Most Four Passive Elements $^{\text {th }}$
}

\author{
Kai Wang ${ }^{\mathrm{a}}$, Michael Z. Q. Chen ${ }^{\mathrm{b}, *}$, Yinlong $\mathrm{Hu}^{\mathrm{a}}$ \\ ${ }^{a}$ School of Automation, Nanjing University of Science and Technology, Nanjing, P.R. China. \\ ${ }^{b}$ Department of Mechanical Engineering, The University of Hong Kong, Pokfulam Road, Hong Kong.
}

\begin{abstract}
In this paper, we consider the passive network synthesis problem of biquadratic impedances with at most four elements, motivated by the passive mechanical control. In order to solve this problem, a necessary and sufficient realizability condition for no more than three elements is obtained by some topological properties derived previously. Furthermore, the constraints on the possible realizations are used to derive the networks which can cover all the cases, and they are classified as several quartets. Through investigating one of the networks in each quartet, we obtain a necessary and sufficient condition for any biquadratic impedance to be realizable with at most four elements. Finally, the interlocking conditions are illustrated graphically, and numerical examples are given.
\end{abstract}

Keywords: Passive network synthesis, biquadratic impedance, inerter, optimization.

\section{Introduction}

The 1930s to the 1970s was the "golden era" of passive network synthesis. Although there have been many elegant results in passive network synthesis [1-4, 20, 27, 28, 50], some fundamental questions still remain unsolved, especially the minimal realization problems in terms of the total number of elements. After the 1970s, interest in passive network synthesis has declined despite some developments in two-port networks [37, 51], positive-real functions [16, 33, 36], and other related areas [34].

Recently, a new mechanical element named inerter has been introduced [40], and the force applied at the two movable terminals is proportional to the acceleration between them. The inerter has been applied to vehicle suspension systems [5, 12, 21, 41, 47], motorcycle steering

\footnotetext{
This paper has appeared in a preliminary form in the Proceedings of the 24th Chinese Control and Decision Conference [45] (in Chinese).

This work is supported by Hong Kong University Committee on Research and Conference Grants under Grant 201111159110, National Natural Science Foundation of China under Grants 61004093, National Key Basic Research Scheme of China ("973 Program") under Grant 2012CB720200, and the "Innovative research projects for graduate students in universities of Jiangsu province” CXLX12_0200.

${ }^{*}$ Corresponding author

Email address: mzqchen@hku.hk (Michael Z. Q. Chen)
} 
instabilities [17], and train suspension systems [25, 47, 49] with performance advantages demonstrated. Furthermore, the inerter completes the analogy between mechanical systems and electrical ones (see [5, 40]). Since then, interest in passive network synthesis has been revived [6-11, 13$15,23,24,46]$, particularly in the mechanical setting as minimal realizations are more essential for mechanical systems. Independently, a renewed attempt on this field has also been advocated by Kalman [26].

This paper is concerned with the minimal realization of biquadratic impedances, which has been widely investigated [8, 19, 23, 24, 29-31, 42-44], but not fully resolved. Recently, Chen and Smith [8] derived a necessary and sufficient condition for any positive-real impedance (admittance) to be realizable with one inductor (spring), one capacitor (inerter), and an arbitrary number of resistors (dampers) without transformers (levers), which is explicitly concerned with entries of a non-negative definite matrix. Through defining 'regularity', [22-24] solved the network realization with five elements and series-parallel networks with six elements. To further simplify the realizations, it is meaningful to obtain a necessary and sufficient condition for any positive-real impedance to be realizable with at most four elements with a corresponding group of covering configurations. Although all the networks having biquadratic impedances with at most five elements have been listed in [29], the method is rather tedious and the realizability conditions of the 108 networks have not been classified in a meaningful way to enlighten further endeavors. Besides, no concise realizability condition with at most four elements has been given in [29]. In this paper, a simpler and more systematic method is used to solve this problem. The results can be used not only for realizing a biquadratic impedance with at most four elements but also for testing whether a five-element realization is minimal or not.

\section{Problem Formulation}

Consider a biquadratic impedance in the form of

$$
Z(s)=\frac{A s^{2}+B s+C}{D s^{2}+E s+F}
$$

where $A, B, C, D, E, F \geq 0$. It is positive-real if and only if $(\sqrt{A F}-\sqrt{C D})^{2} \leq B E[10,18]$. Any positive-real biquadratic impedance (1) with any of its six parameters equal to zero can be realized by a series-parallel network with at most two reactive elements and two resistive elements through the Foster preamble [22, Lemma 8]. Hence, to investigate realizations with at most four elements, it suffices to consider $A, B, C, D, E, F>0$, which we assume here. The resultant of (1) is

$$
K:=(A F-C D)^{2}-(A E-B D)(B F-C E) .
$$

There exist common factors between the numerator and denominator of (1) if and only if $K=0$. A systematic method will be used to find a necessary and sufficient condition for $Z(s)$ to be realizable with at most four elements. In this method, a concept named quartet [22] is introduced to classify the networks that cover the conditions. The networks considered in this paper contain no more than three kinds of elements, which are resistors, capacitors, and inductors. 


\section{Main Results}

In this section, through presenting a series of constraints (Lemma 1-2, Lemma 4-5), covering networks are presented and classified (Theorem 1). Summarizing their realizability conditions (Theorem 2-4), the final condition (Theorem 5) is presented. Consider any one-port network $N$ whose two terminals are denoted as $a$ and $a^{\prime}$. If we regard each element as an edge, then the network graph $\mathcal{G}_{e}$ is formed. $\mathcal{C}\left(a, a^{\prime}\right)$ denotes the cut-set [39] which separates $\mathcal{G}_{e}$ into two connected subgraphs $\mathcal{G}_{1}$ and $\mathcal{G}_{2}$ containing $a$ and $a^{\prime}$, respectively. $\mathcal{P}\left(a, a^{\prime}\right)$ denotes the path [39] whose end vertices are $a$ and $a^{\prime}$.

Lemma 1. Consider a biquadratic impedance $Z(s)$ in the form of (1) where $A, B, C, D, E, F$ $>0$. For any network $N$ with two terminals $a$ and $a^{\prime}$ that can realize $Z(s)$, its network graph does not contain any path $\mathcal{P}\left(a, a^{\prime}\right)$ or cut-set $\mathcal{C}\left(a, a^{\prime}\right)$ whose edges correspond to only one kind of reactive elements.

Proof. Assume that this kind of path $\mathcal{P}\left(a, a^{\prime}\right)$ or cut-set $\mathcal{C}\left(a, a^{\prime}\right)$ exists. Then it is known from [38] that the impedance of $N$ must contain zeros or poles at $s=0$ or $s=\infty$, which contradicts with the assumption.

More generally, we have the following lemma.

Lemma 2. Any biquadratic impedance $Z(s)$ in the form of (1) with $A, B, C, D, E, F>0$ cannot be realized by the networks whose structures are shown in Fig. 1.

Proof. From [22], we know that there exist poles for the impedance of Fig. 1(a) and zeros for the that of Fig. 1(b) at $s=j \omega$ or $s=\infty$, implying that some of the coefficients must be zero. Hence, the lemma is proved.

Lemma 3. Consider a biquadratic impedance $Z(s)$ in the form of (1), where $A, B, C, D, E, F$ $>0$. Then it can be realized with at most three elements if and only if $K=0$.

Proof. Sufficiency. Since $K=0, Z(s)$ reduces to $Z(s)=\left(a_{0} s+a_{1}\right) /\left(d_{0} s+d_{1}\right)$, where $a_{0}, a_{1}, d_{0}$, $d_{1}>0$. It can be verified that $Z(s)$ is realizable by the network in Fig. 2 by the Foster preamble when $a_{0} d_{1}-a_{1} d_{0} \neq 0$, or by just a resistor when $a_{0} d_{1}-a_{1} d_{0}=0$.

Necessity. By the principle of duality, only network graphs shown in Fig. 3 need to be discussed. For Fig. 3(a), the only edge should correspond to the resistor, otherwise it will result in a path $\mathcal{P}\left(a, a^{\prime}\right)$ or a cut-set $\mathcal{C}\left(a, a^{\prime}\right)$ corresponding to one kind of reactive elements, which is impossible by Lemma 1. For Fig. 3(b), Fig. 3(c), and Fig. 3(d), to avoid repetition, the networks that can be equivalent to the one containing fewer elements are not considered, as the discussion is in the order of the increasing number of elements from Fig. 3(a) to Fig. 3(d). Furthermore, by Lemma 1 and Lemma 2, the network graphs in Fig. 3(b) and Fig. 3(c) are directly eliminated, and Edge 1 and only one of Edge 2 or Edge 3 of the network graph in Fig. 3(d) correspond to resistors, yielding networks in Fig. 2(a) and Fig. 2(c). By the principle of duality, we obtain Fig. 2(b) and Fig. 2(d). It is calculated that impedances of these networks satisfy $K=0$.

In the remaining discussion, we only need to consider $K \neq 0$. 


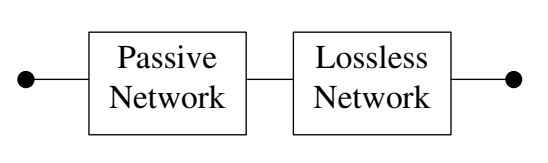

(a)

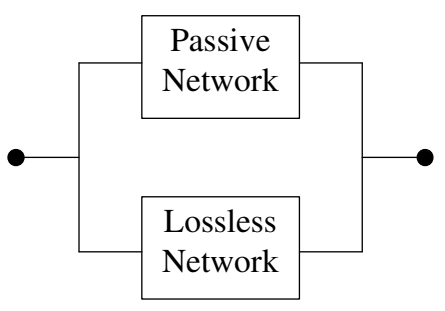

(b)

Figure 1: The network structures that a lossless subnetwork and any passive one are (a) in series or (b) in parallel.

Lemma 4. Consider a biquadratic impedance $Z(s)$ in the form of (1), where $A, B, C, D, E, F$ $>0$ and $K \neq 0$. If $Z(s)$ can be realized by the network $N$ with four elements, there do not exist two elements of the same kind in series or in parallel.

Proof. Assume such two elements exist, then the network $N$ can be equivalent to the one with at most three elements, implying $K=0$ by Lemma 3 . Thus, this lemma is proved.

Lemma 5. Consider a biquadratic impedance $Z(s)$ in the form of (1) where $A, B, C, D, E, F$ $>0$, and $K \neq 0$. If it can be realized with four elements, the number of reactive elements must be either two or three.

(a)
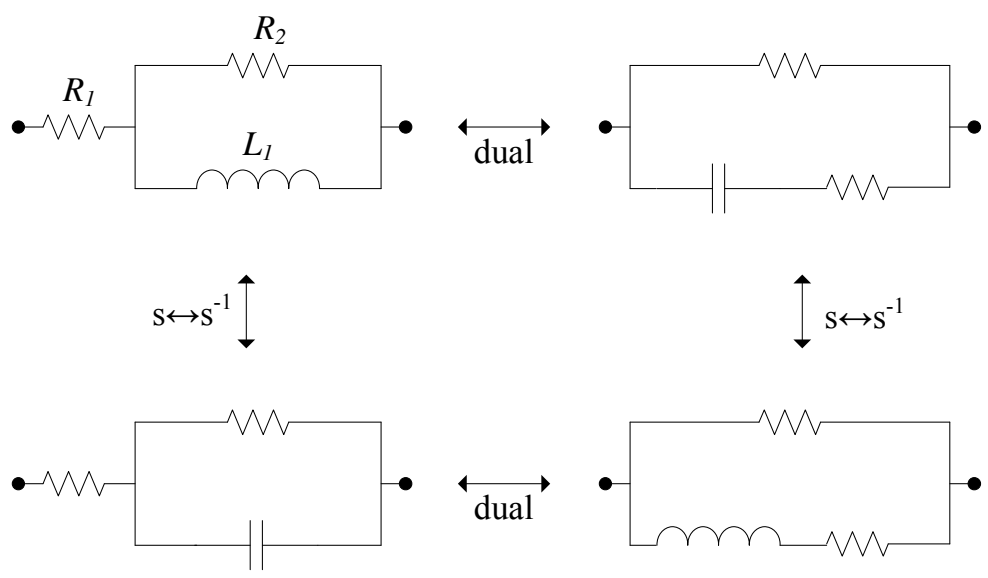

(c) (d)

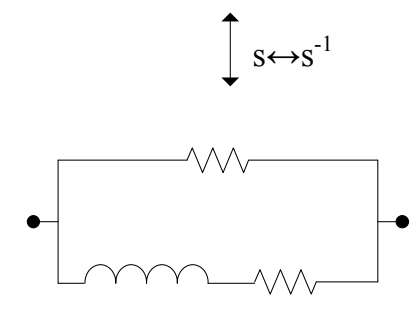

(b)

Figure 2: The quartet of three-element networks discussed in Lemma 3.

Proof. Assume that there is at most one reactive element, then it is implied from [1] that $Z(s)$ can be expressed as a bilinear function whose McMillan degree is one, implying that $K=0$. Assume that the network contains four reactive elements, then all the poles of $Z(s)$ must be at $s=j \omega$ or $s=\infty$ [20], which contradicts with the fact that all the coefficients are positive. Thus the lemma is proved. 


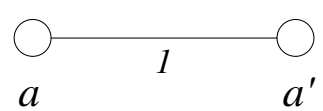

(a)

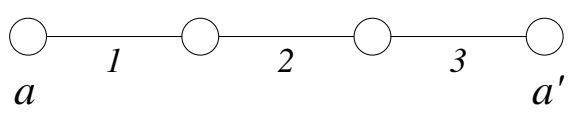

(c)

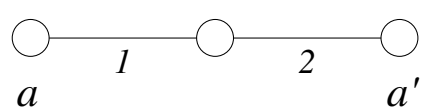

(b)

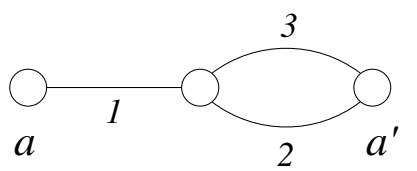

(d)

Figure 3: The network graphs of the networks with at most three elements (one half).

Theorem 1. Consider a biquadratic impedance $Z(s)$ in the form of (1), where $A, B, C, D, E, F$ $>0$ and $K \neq 0$. Then $Z(s)$ can be realized with four elements if and only if it is the impedance of at least one of the networks in the quartets in Fig. 4, Fig. 5, and Fig. 6.

Proof. Necessity. By the principle of duality, only the five network graphs in Fig. 7 need to be considered. First, the one in Fig. 7(a) is directly eliminated by Lemma 4 for that at least two elements of the same kind must be in series. For Fig. 7(b), Edge 1 and Edge 2 must both correspond to resistors by Lemma 1 , otherwise a cut-set $\mathcal{C}\left(a, a^{\prime}\right)$ whose edges correspond to only one kind of reactive elements must exist. Hence, Fig. 7(b) is eliminated by Lemma 4. Similarly, Edge 1 of Fig. 7(c) and Fig. 7(d) should also correspond to resistors by Lemma 1. Furthermore,

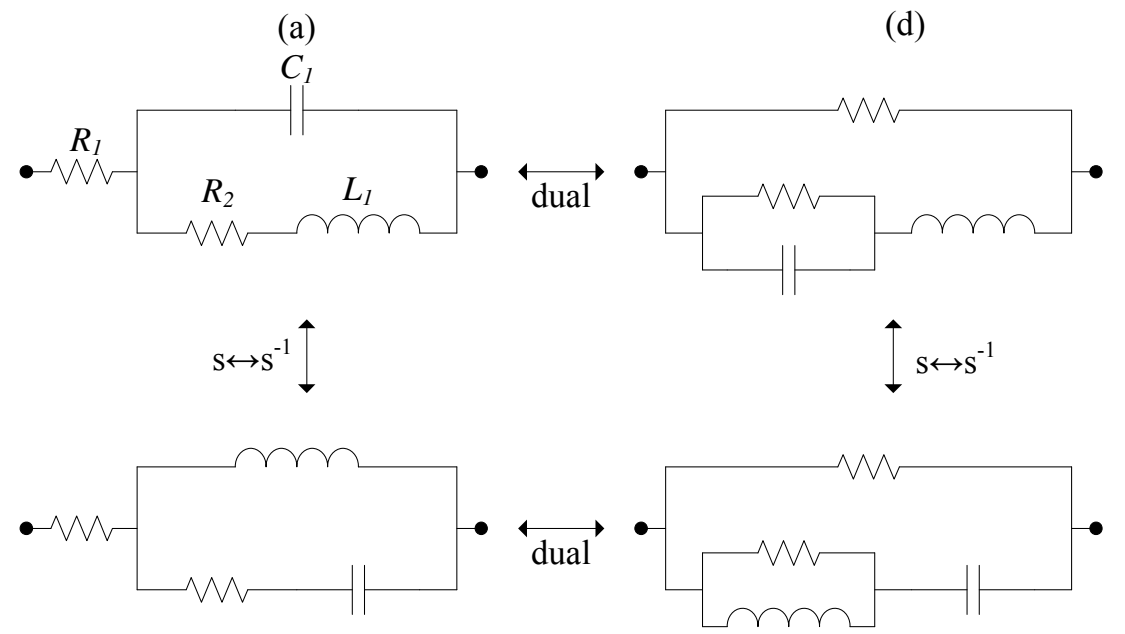

(c)

(b)

Figure 4: First network quartet discussed in Theorem 1.

there are at most two reactive elements among Edge 2, Edge 3, and Edge 4 by Lemma 2. Together with Lemma 5, we assert that the number is exactly two. Besides, for Fig. 7(c), the two reactive elements cannot be of the same kind. Otherwise, a cut-set $\mathcal{C}\left(a, a^{\prime}\right)$ whose edges correspond to 


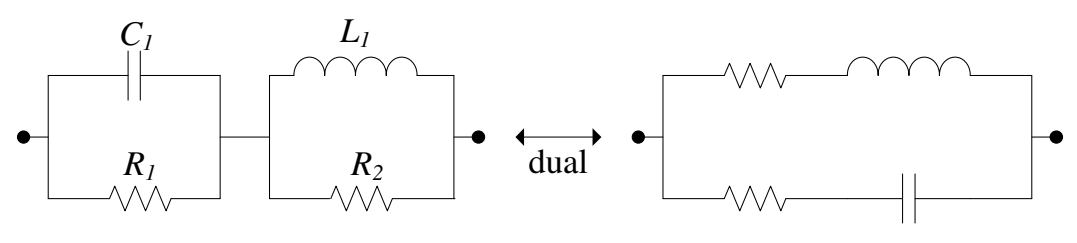

(a)

(b)

Figure 5: Second network quartet discussed in Theorem 1.

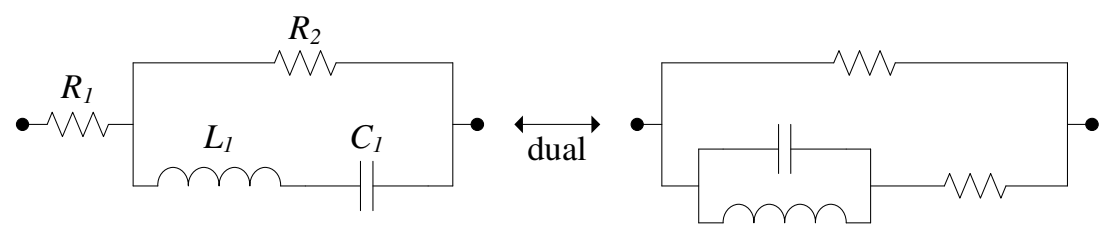

(a)

(b)

Figure 6: Third network quartet discussed in Theorem 1.

only one type of reactive elements exists, contradicting with the assumption by Lemma 1, or there exist two elements of the same kind in series, which is also impossible by Lemma 4. Therefore, the only possible networks are shown in Fig. 4(a), Fig. 4(c) and Fig. 6(a). Then the principle of duality yields quartets in Fig. 4 and Fig. 6. For Fig. 7(d), the two reactive elements cannot be of the same kind by Lemma 4. The only possible network is shown in Fig. 8(b), which further results in the quartet in Fig. 8 by the principle of duality. Finally, we discuss Fig. 7(e). There is one resistor in each of the two subnetworks in series by Lemma 2, and the remaining two reactive elements cannot be of the same kind by Lemma 1 . Therefore, the only possible network is shown in Fig. 5(a), which yields the quartet in Fig. 5 by the principle of duality. Finally, by the equivalence discussed in [32], we see the quartets in Fig. 6 and Fig. 8 are essentially equivalent. Hence, the networks in Fig. 4, Fig. 5, and Fig. 6 can cover all the possible cases.

Sufficiency. It is noted that all of the networks in Fig. 4, Fig. 5, and Fig. 6 contain four elements. Hence, if a given $Z(s)$ in the form of (1), where $A, B, C, D, E, F>0$ and $K \neq 0$ is realizable by one of them, then $Z(s)$ can indeed be realized with four elements.

Next, we investigate the realizability conditions of the networks in these three quartets in order to obtain the final conditions. Although the conditions of these networks have been shown in [29], the derivation processes were not presented. For each quartet, we only need to discuss one network, as realizability conditions of other networks can be immediately obtained by the principle of duality or $s \leftrightarrow s^{-1}$.

Theorem 2. Consider a biquadratic impedance $Z(s)$ in the form of (1), where $A, B, C, D, E, F$ $>0$ and $K \neq 0$. Then it can be realized by the network in Fig. 4(a) if and only if

$$
\begin{aligned}
A F & <C D \\
A E^{2}+C D^{2} & =A D F+B D E .
\end{aligned}
$$




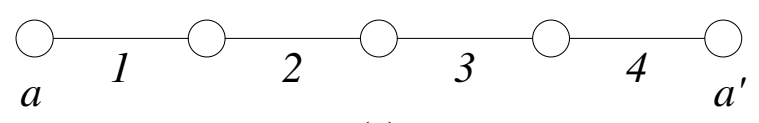

(a)

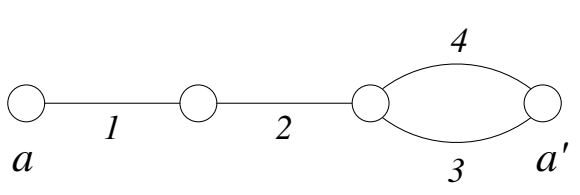

(b)

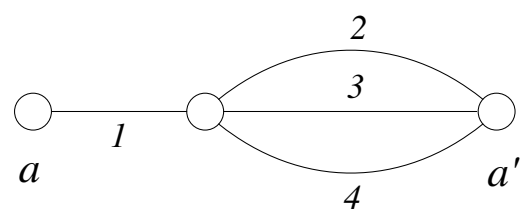

(d)

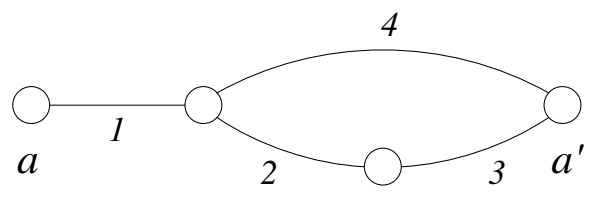

(c)

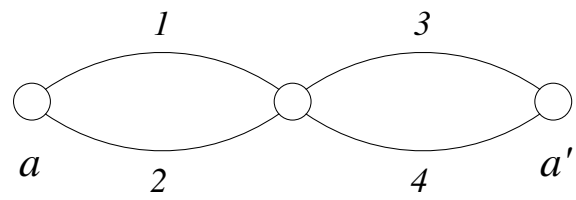

(e)

Figure 7: The network graphs of the networks with at most four elements (one half).

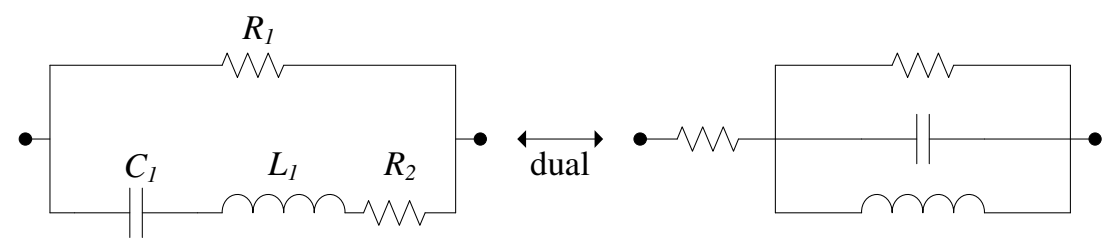

(a)

(b)

Figure 8: Network quartet which is equivalent to that in Fig. 6.

Furthermore, if the condition is satisfied, the values of the elements can be expressed as

$$
R_{1}=\frac{A}{D}, R_{2}=\frac{C D-A F}{D F}, L_{1}=\frac{C D-A F}{E F}, C_{1}=\frac{D E}{C D-A F} .
$$

Proof. The proof is presented in Appendix.

Theorem 3. Consider a biquadratic impedance $Z(s)$ in the form of (1) where $A, B, C, D, E, F$ $>0$, and $K \neq 0$. Then it can be realized by the network as shown in Fig. 5(a) if and only if

$$
(A F+C D)^{2}-B E(A F+C D)+B^{2} D F=0 .
$$

Furthermore, if the condition is satisfied, then the values of the elements can be expressed as

$$
R_{1}=\frac{C}{F}, R_{2}=\frac{A}{D}, L_{1}=\frac{A B}{A F+C D}, C_{1}=\frac{A F+C D}{B C} .
$$


Proof. The proof is presented in Appendix.

Theorem 4. Consider a biquadratic impedance $Z(s)$ in the form of (1) where $A, B, C, D, E, F$ $>0$, and $K \neq 0$. Then it can be realized by the network as shown in Fig. 6(a) if and only if

$$
\begin{aligned}
& A F=C D, \\
& B F<C E .
\end{aligned}
$$

Furthermore, if the condition is satisfied, then the values of the elements can be expressed as

$$
R_{1}=\frac{B}{E}, R_{2}=\frac{C E-B F}{E F}, L_{1}=\frac{A(C E-B F)}{C E^{2}}, C_{1}=\frac{E^{2}}{C E-B F} .
$$

Proof. The proof is presented in Appendix.

The realizability conditions of other networks in Fig. 4, Fig. 5, and Fig. 6 can be directly derived from Fig. 4(a), Fig. 5(a), and Fig. 6(a) by exchanging the coefficients properly, and networks of these three quartets are summarized in Table 1 . The realizability condition of the dual network of $N$ is obtained from that of $N$ through $A \leftrightarrow D, B \leftrightarrow E$, and $C \leftrightarrow F$; the realizability condition of the network that is $s \leftrightarrow s^{-1}$ with $N$ is obtained from that of $N$ through $A \leftrightarrow C$ and $D \leftrightarrow F$.

Theorem 5. Consider a biquadratic impedance $Z(s)$ in the form of (1), where $A, B, C, D, E, F$ $>0$. Then it can be realized with at most four elements if and only if at least one of the following conditions holds

(a) $K=0$;

(b) $A F=C D$;

(c) $A F<C D$, and $\left\{A E^{2}+C D^{2}=A D F+B D E\right\} \cup\left\{F B^{2}+D C^{2}=A C F+B C E\right\}$;

(d) $A F>C D$, and $\left\{C E^{2}+A F^{2}=C D F+B E F\right\} \cup\left\{D B^{2}+F A^{2}=A C D+A B E\right\}$;

(e) $(A F+C D)^{2}+B^{2} D F=B E(A F+C D)$, or $(A F+C D)^{2}+E^{2} A C=B E(A F+C D)$.

Proof. Combining Lemma 3, Theorem 1, and Table 1, this theorem can be proved.

\section{Graphical Illustration of the Conditions}

To illustrate the realizability conditions more intuitively, we now express them graphically. Hence, the following canonical expression used in [22] is introduced as

$$
Z_{c}(s)=\frac{s^{2}+2 U \sqrt{W} s+W}{s^{2}+(2 V / \sqrt{W}) s+1 / W},
$$

where $W=\sqrt{C D /(A F)}, U=B /(2 \sqrt{A C})$, and $V=E /(2 \sqrt{D F})$. It can be seen that $Z_{c}(s)$ can be obtained from $Z(s)$ by $Z_{c}(s)=\alpha Z(\beta s)$, where $\alpha=D / A$, and $\beta=\sqrt[4]{C F /(A D)}$. Hence $Z_{c}(s)$ can be realized by all the networks that can realize $Z(s)$ by properly changing the values of the elements, and vice versa. 


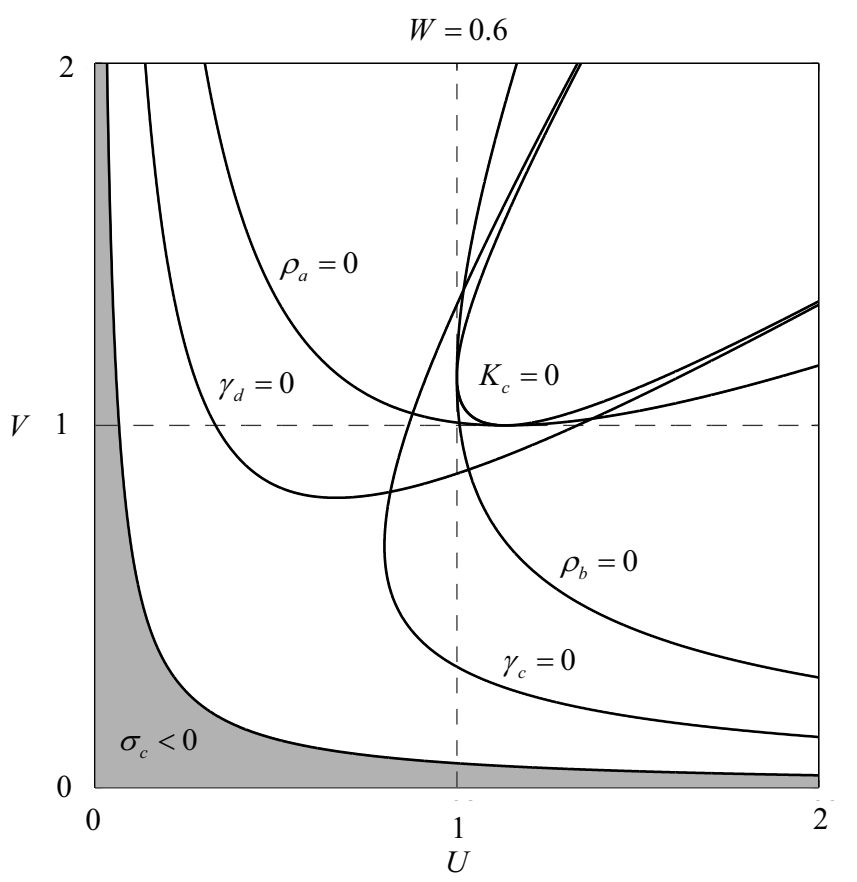

(a)

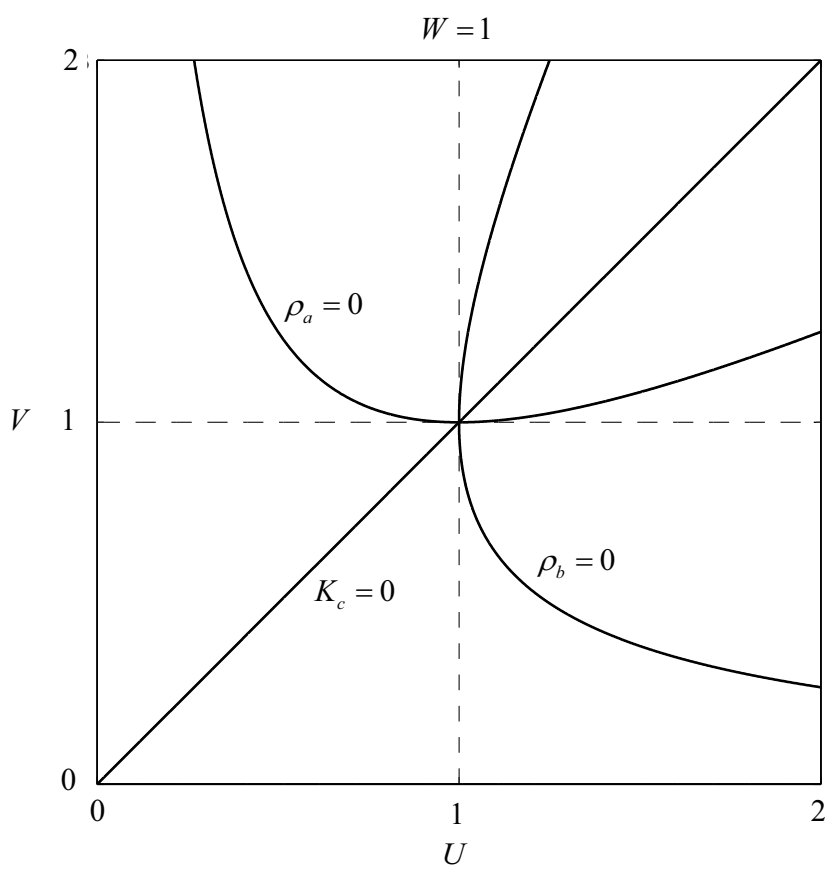

(b)

Figure 9: The conditions for impedance (10) to be realizable with four elements ((a) for $W=0.6$; (b) for $W=1$ ). 
Table 1: Necessary and sufficient conditions for the biquadratic impedance in the form (1) to be realizable by the networks shown in Fig. 4, Fig. 5, and Fig. 6, where the second columns provide the number of the networks in [29].

\begin{tabular}{|l||c|c|}
\hline Network & {$[29]$} & Realizability Condition \\
\hline Fig. 4(a) & 87 & $A F<C D$, and $A E^{2}+C D^{2}=A D F+B D E$ \\
\hline Fig. 4(b) & 88 & $A F<C D$, and $F B^{2}+D C^{2}=A C F+B C E$ \\
\hline Fig. 4(c) & 63 & $C D<A F$, and $C E^{2}+A F^{2}=C D F+B E F$ \\
\hline Fig. 4(d) & 62 & $C D<A F$, and $D B^{2}+F A^{2}=A C D+A B E$ \\
\hline Fig. 5(a) & 96 & $(A F+C D)^{2}-B E(A F+C D)+B^{2} D F=0$ \\
\hline Fig. 5(b) & 97 & $(A F+C D)^{2}-B E(A F+C D)+E^{2} A C=0$ \\
\hline Fig. 6(a) & 71 & $A F=C D$, and $B F<C E$ \\
\hline Fig. 6(b) & 74 & $A F=C D$, and $C E<B F$ \\
\hline
\end{tabular}

Denote that

$$
\begin{aligned}
& \gamma_{a}(U, V, W)=4 V^{2}+W^{2}-4 W V U-1, \\
& \gamma_{b}(U, V, W)=\gamma_{a}(V, U, W), \\
& \gamma_{c}(U, V, W)=\gamma_{a}\left(U, V, W^{-1}\right), \\
& \gamma_{d}(U, V, W)=\gamma_{a}\left(V, U, W^{-1}\right), \\
& \rho_{a}(U, V, W)=(W+1 / W)^{2}-4 U V(W+1 / W)+4 U^{2}, \\
& \rho_{b}(U, V, W)=\rho_{a}(V, U, W)=\rho_{a}\left(V, U, W^{-1}\right) .
\end{aligned}
$$

Let

$$
K_{c}:=4 U^{2}+4 V^{2}-4 U V(W+1 / W)+(W-1 / W)^{2} .
$$

By using the relations: $W=\sqrt{C D /(A F)}, U=B /(2 \sqrt{A C})$, and $V=E /(2 \sqrt{D F})$, the condition of Theorem 5 can be equivalent to at least one of the five conditions holds:

(a) $K_{c}=0$;

(b) $W=1$;

(c) $W>1$;

(d) $W<1$, and $(U, V, W) \in\left\{(U, V, W) \mid \gamma_{c}(U, V, W)=0 \cup \gamma_{d}(U, V, W)=0\right\}$;

(e) $(U, V, W) \in\left\{(U, V, W) \mid \rho_{a}(U, V, W)=0 \cup \rho_{b}(U, V, W)=0\right\}$.

Besides, $Z_{c}(s)$ is positive-real if and only if

$$
\sigma_{c}=4 U V+2-(1 / W+W) \geq 0 .
$$

The $U-V$ plane is shown in Fig. 9 for fixed $W$. Fig. 9(a) shows the condition when $W=0.6$, representing the case of $W>1$. The condition is graphically illustrated by five curves that intersect at several points, which means that Condition (a), Condition (d), and Condition (e) have overlap only at some special cases. Fig. 9(b) shows the realizability condition when $W=1$. The figure of the case where $W>1$ is similar to $W<1$, which is not shown for brevity. 


\section{Numerical Examples}

Two numerical examples of realization are provided as follows. Consider the biquadratic impedance $Z(s)$ in the form of (1). If $A=1, B=1, C=3, D=1, E=53 / 7$, and $F=4$, then it is calculated that $K=6075 / 49>0$, and $(A F+C D)^{2}+B^{2} D F=B E(A F+C D)=53$. Hence, $Z(s)$ is realizable as in Fig. 5 with $R_{1}=3 / 4 \Omega, R_{2}=1 \Omega, L_{1}=1 / 7 H$, and $C_{1}=7 / 3 F$. If $A=1, B=2, C=1, D=1, E=1$, and $F=2$, then it is calculated that $K=4>0$ $A F-C D=1>0, C E^{2}+A F^{2}-C D F-B E F=-1<0, D B^{2}+F A^{2}-A C D-A B E=3>0$, $(A F+C D)^{2}+B^{2} D F-B E(A F+C D)=11>0$, and $(A F+C D)^{2}+E^{2} A C-B E(A F+C D)=$ $4>0$, which does not satisfy the condition of Theorem 5 . Hence, it cannot be realized with at most four elements. But it can be realized by a five-element network in [23, Fig. 8] with $R_{1}=1 / 2 \Omega$, $R_{2}=1 / 2 \Omega, R_{3}=4 \Omega, L_{1}=3 / 4 H$, and $C_{1}=3 / 4 F$, which is consequently a minimal realization of $Z(s)$ in terms of the total number of elements.

Moreover, in order to illustrate the practical meaning of this paper, two examples are provided to consider the performances of vehicle suspension systems. Consider the quarter car vehicle model shown in Fig. 10, where the parameters are chosen as $m_{s}=250 \mathrm{~kg}, m_{u}=35 \mathrm{~kg}, k_{t}=$ $150 \mathrm{kN} / \mathrm{m}$. For brevity, only the ride comfort performance measure $J_{1}$ defined in $[35,48]$ is investigated.

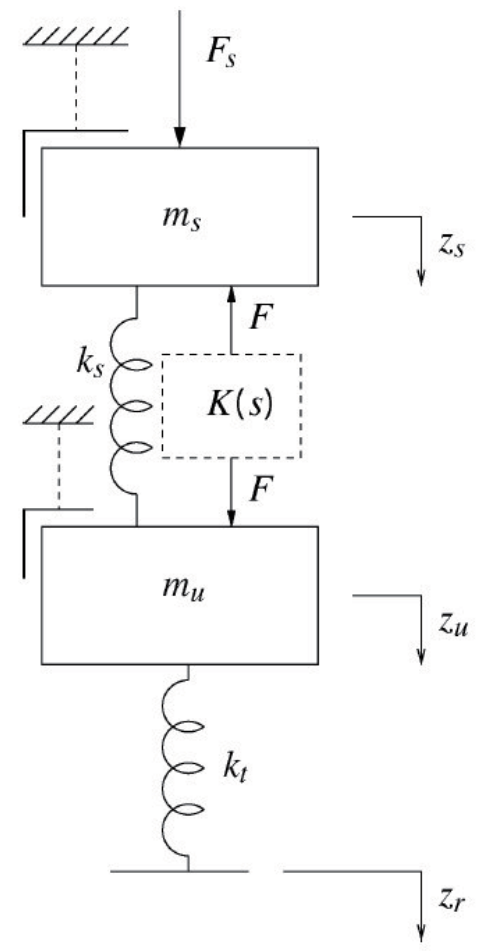

Figure 10: Quarter-car vehicle model with predetermined static stiffness.

The first example is carried out based on the results in [35], where the $K(s)$ in Fig. 10 denotes the passive mechanical networks consisting of finite interconnections of springs, dampers and inerters. In [35], there is no restriction on the number of element, while in this paper, at most four 
elements are permitted. The simulation results are shown in Fig. 11 and Fig. 12, where we see that the performance $J_{1}$ is slightly degraded by restricting the maximum number of element to be four. It is shown in Fig. 12 that at most 3.5\% degradation is obtained compared with the results in [35].

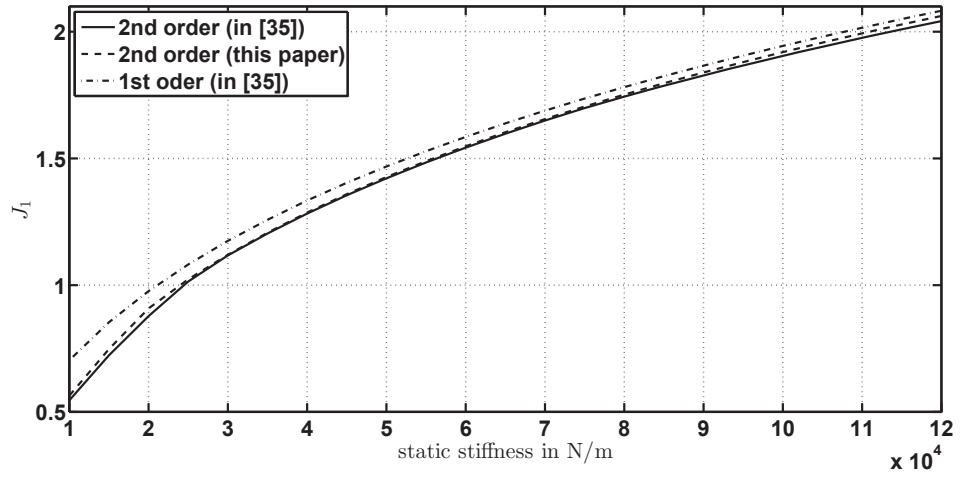

Figure 11: Comparison of the $J_{1}$ performance with the results in [35].

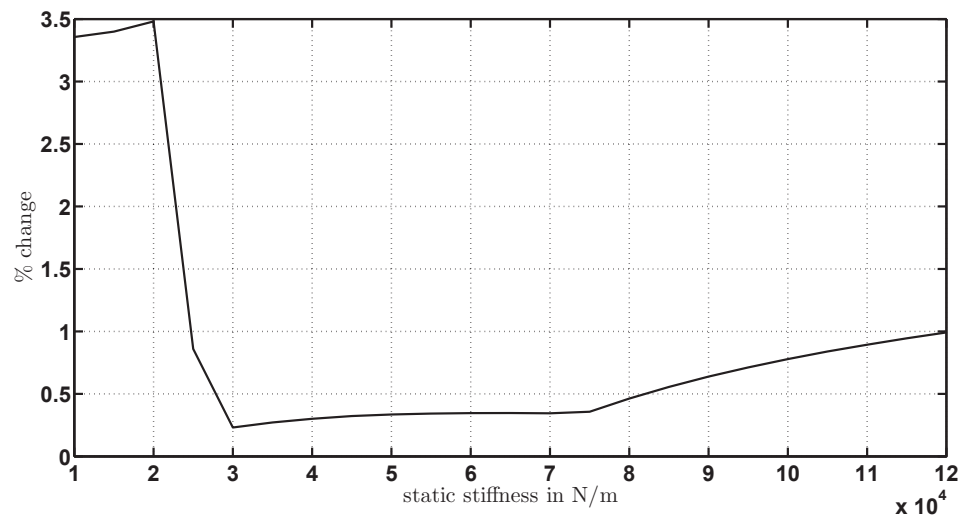

Figure 12: Performance degradation compared with [35].

The first example indicates that the performance will be degraded if the number of elements is restricted to be at most four, while in the second example, we will show another situation where the performance is almost not affected by the restriction but less-complex realization will be achieved. In the second example, the mechatronic network strut LMIS3 in [48] is considered, where the electrical circuits in the mechatronic network strut are assumed to be biquadratic. Similarly, in this paper, at most four elements are permitted to realize these biquadratic impedance electrical circuits. The same parameters in [48] are employed and the simulation results are shown in Fig. 13, where we see that the results obtained in this paper is almost the same as those in [48]. Taking $k_{s}=50 \mathrm{kN} / \mathrm{m}$ for example, the optimal $J_{1}$ in [48] is 1.324 , while the one in this paper is 1.325 with only $0.08 \%$ degradation. However, the optimal electrical impedance in [48] when $k_{s}=50$ $\mathrm{kN} / \mathrm{m}$ is

$$
\frac{1.665 \times 10^{5} s^{2}+5.776 \times 10^{5} s+5.466 \times 10^{7}}{s^{2}+1.544 \times 10^{2} s+0.342},
$$


which does not satisfy the conditions in Theorem 5, hence it cannot be realized with four elements. The minimal realization given in [48] is shown in Fig. 14, where six elements are employed.

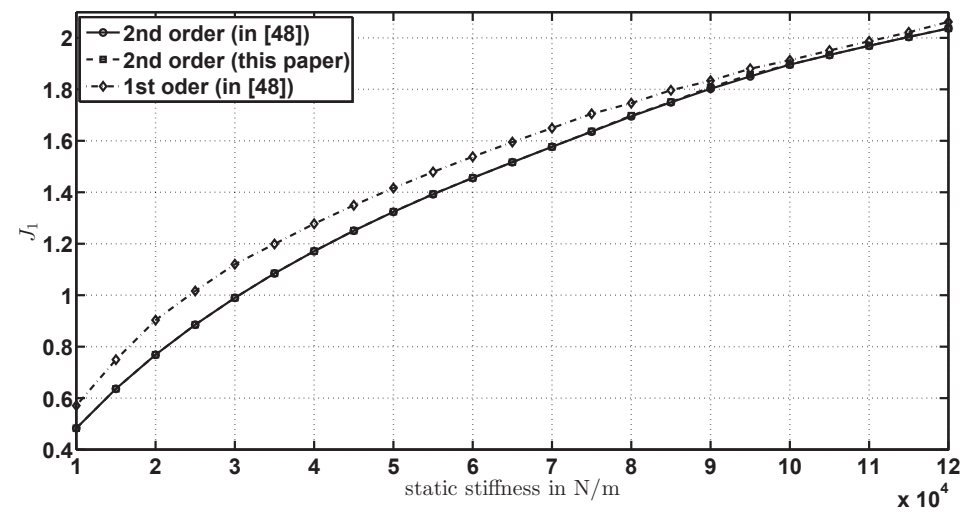

Figure 13: Comparison of the $J_{1}$ performance with the results in [48].

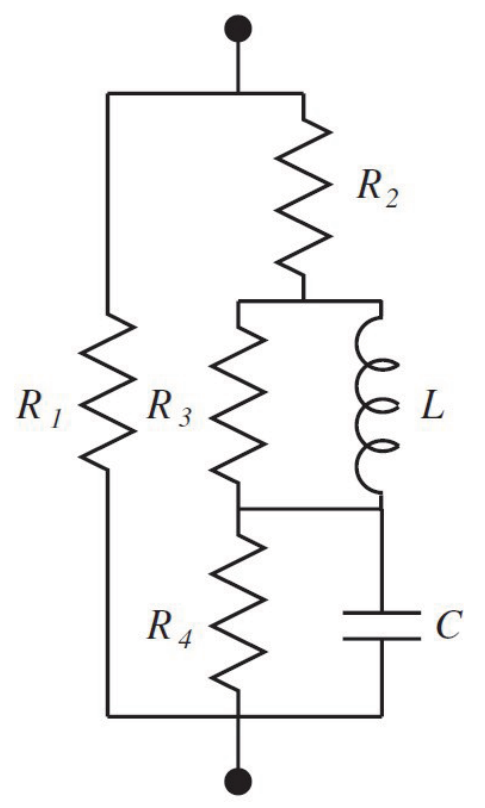

Figure 14: Optimal biquadratic impedance realized in [48].

The optimal impedance in this paper is

$$
\frac{1429.280 s^{2}+4684.116 s+4.695 \times 10^{5}}{s^{2}+1.323 \times 10^{4} s+328.477},
$$

which satisfies the condition in Theorem 4 , hence it can be realized by the network as shown in Fig. 6(a) with $R_{1}=0.354 \Omega, R_{2}=1.429 \times 10^{3} \Omega, L_{1}=0.108 \mathrm{H}, C_{1}=0.0282 \mathrm{~F}$. The coefficients for other components of LMIS3 in this paper are $k_{b}=5.340 \times 10^{4} \mathrm{~N} / \mathrm{m}, c_{b}=4.079 \times 10^{5}$ 
$\mathrm{Ns} / \mathrm{m}, b_{m}=28 \mathrm{~kg}, c_{m}=0 \mathrm{Ns} / \mathrm{m}$, which have similar values as those in [48]. This example effectively demonstrates that although the system performance would be slightly degraded, simpler realizations can be achieved by applying the conditions in this paper.

\section{Conclusion}

This paper has investigated the synthesis problem of biquadratic impedances with at most four elements, motivated by the passive mechanical control. A necessary and sufficient condition was obtained for a biquadratic impedance to be realizable with at most three elements. Furthermore, the four-element networks that cannot be reduced to those with less elements were discussed. The networks covering all the possibilities were presented and classified as three quartets. A series of constraints were presented to simplify the search. After deriving the realizability conditions of these networks, a necessary and sufficient condition for the biquadratic impedance to be realizable with at most four elements was obtained. Finally, the conditions were intuitively illustrated in graphs, and some numerical examples were given. The results obtained can contribute to the minimal realization of biquadratic impedances.

\section{Appendix}

\section{Proof of Theorem 2}

Necessity. The impedance of the network in Fig. 4(a) can be calculated as

$$
Z(s)=\frac{R_{1} C_{1} L_{1} s^{2}+\left(R_{1} R_{2} C_{1}+L_{1}\right) s+\left(R_{1}+R_{2}\right)}{C_{1} L_{1} s^{2}+R_{2} C_{1} s+1} .
$$

Then we obtain

$$
\begin{aligned}
R_{1} C_{1} L_{1} & =k A, \\
R_{1} R_{2} C_{1}+L_{1} & =k B, \\
R_{1}+R_{2} & =k C, \\
C_{1} L_{1} & =k D, \\
R_{2} C_{1} & =k E, \\
1 & =k F,
\end{aligned}
$$

where $k>0$. (17) is equivalent to

$$
k=\frac{1}{F} .
$$

From (12) and (15), we can obtain the expression of $R_{1}$. Substituting (18) and the expression of $R_{1}$ into (14), we obtain $R_{2}$, implying Condition (2). Substituting (18) and the expression of $R_{2}$ into (16), we obtain $C_{1}$. Finally, from (12) and (13), we obtain the expression of $L_{1}$ and Condition (3). 
Sufficiency. Calculate $R_{1}, R_{2}, L_{1}$, and $C_{1}$ by (4), and $k$ from (18), which are all positive and finite by Condition (2). Furthermore, (12)-(17) must hold by Condition (3). Therefore, the biquadratic impedance $Z(s)$ in the form of (1) can be realized by the network in Fig. 4(a) with the values of the elements being expressed as (4).

\section{Proof of Theorem 3}

Necessity. The impedance of the network shown in Fig. 5(a) can be calculated to be

$$
Z(s)=\frac{R_{1} R_{2} L_{1} C_{1} s^{2}+L_{1}\left(R_{1}+R_{2}\right) s+R_{1} R_{2}}{R_{1} L_{1} C_{1} s^{2}+\left(L_{1}+R_{1} R_{2} C_{1}\right) s+R_{2}} .
$$

Suppose that it can realize the biquadratic impedance in the form of (1) where $A, B, C, D, E, F$ $>0$, and $K \neq 0$. Then we obtain

$$
\begin{array}{r}
R_{1} R_{2} L_{1} C_{1}=k A, L_{1}\left(R_{1}+R_{2}\right)=k B, R_{1} R_{2}=k C, \\
R_{1} L_{1} C_{1}=k D, L_{1}+R_{1} R_{2} C_{1}=k E, R_{2}=k F,
\end{array}
$$

where $k>0$. From the third and sixth equation of (20), we obtain $R_{1}$. Then the second equation is equivalent to $L_{1}=k B /\left(R_{1}+R_{2}\right)=k B F /\left(C+k F^{2}\right)$, and the fourth one is equivalent to $C_{1}=k D /\left(R_{1} L_{1}\right)=D\left(C+k F^{2}\right) /(B C)$. Substituting all these into the first equation, $k$ is calculated as $k=A /(D F)$, resulting in the expressions of $R_{2}, L_{1}$, and $C_{1}$. Finally, together with the fifth equation of (20), we obtain Condition (5).

Sufficiency. Calculate $R_{1}, R_{2}, L_{1}$, and $C_{1}$ by (6), and $k$ as $k=A /(D F)$, which are all positive and finite by Condition (5). Furthermore, it is calculated that (20) must hold by Condition (5). Therefore, the biquadratic impedance $Z(s)$ in the form of (1) can be realized by the network as shown in Fig. 5(a) with the values of the elements being expressed as (6).

\section{Proof of Theorem 4}

Necessity. The impedance of the network shown in Fig. 6(a) is calculated to be

$$
Z(s)=\frac{L_{1} C_{1}\left(R_{1}+R_{2}\right) s^{2}+R_{1} R_{2} C_{1} s+\left(R_{1}+R_{2}\right)}{L_{1} C_{1} s^{2}+R_{2} C_{1} s+1} .
$$

Then we obtain

$$
\begin{gathered}
L_{1} C_{1}\left(R_{1}+R_{2}\right)=k A, R_{1} R_{2} C_{1}=k B, R_{1}+R_{2}=k C, \\
L_{1} C_{1}=k D, \quad R_{2} C_{1}=k E, \quad 1=k F,
\end{gathered}
$$

where $k>0$. The sixth equation of (22) is equivalent to $k=1 / F$. From the first and third equation, we obtain $L_{1} C_{1}=A / C$. Together with the fourth equation (22), we obtain $k=A /(C D)$, implying that Condition (7) must hold. Furthermore, the expressions of $R_{1}, R_{2}, L_{1}$, and $C_{1}$ can be solved, implying the satisfaction of Condition (8).

Sufficiency. Calculate $R_{1}, R_{2}, L_{1}$, and $C_{1}$ by (9), and $k$ as $k=1 / F$, which are all positive and finite by Conditions (7)-(8). Furthermore, it is calculated that (22) must hold by Condition (7). Therefore, the biquadratic impedance $Z(s)$ in the form of (1) can be realized by the network as shown in Fig. 6(a) with the values of the elements being expressed as (9). 


\section{ACKNOWLEDGMENT}

The authors are grateful to the Associate Editor and the reviewers for their insightful suggestions.

\section{References}

[1] B. D. O. Anderson, S. Vongpanitlerd, Network Analysis and Synthesis: A Modern Systems Theory Approach, NJ: Prentice Hall, 1973.

[2] J. Bertrand, C. C Halkias, Analysis and synthesis of resistive n-port networks, Journal of the Franklin Institute 295 (1973) 451-467.

[3] T. A. Bickart, D. W. Melvin, Synthesis of active RC multiport networks with grounded ports, Journal of the Franklin Institute 294 (1972) 289-312.

[4] R. Bott, R. J. Duffin, Impedance synthesis without use of transformers, J. Appl. Phys. 20 (1949) 816.

[5] M. Z. Q. Chen, C. Papageorgiou, F. Scheibe, F.-C. Wang, M. C. Smith, The missing mechanical circuit element, IEEE Circuits Syst. Mag. 9 (2009) 10-26.

[6] M. Z. Q. Chen and M. C. Smith, Mechanical Networks Comprising One Damper and One Inerter Engineering Department, Cambridge University , Cambridge, U.K., Tech. Rep. CUED/F-INFENG/TR.569, 2006.

[7] M. Z. Q. Chen, Passive Network Synthesis of Restricted Complexity, Ph.D. dissertation, University of Cambridge, Cambridge, UK, 2007.

[8] M. Z. Q. Chen, M. C. Smith, Electrical and mechanical passive network synthesis, in Recent Advances in Learning and Control, Lecture Notes in Control and Information Sciences, V. D. Blondel, S. P. Boyd, and H. Kimura, Eds., New York: Springer-Verlag, 371 (2008) 35-50.

[9] M. Z. Q. Chen, A note on PIN polynomials and PRIN rational functions, IEEE Trans. Circuits and Systems-II: Express Briefs 55 (2008) 462-463.

[10] M. Z. Q. Chen, M. C. Smith, A note on tests for positive-real functions, IEEE Trans. Automat. Control 54 (2009) 390-393.

[11] M. Z. Q. Chen, M. C. Smith, Restricted complexity network realizations for passive mechanical control, IEEE Trans. Automat. Control 54 (2009) 2290-2301.

[12] M. Z. Q. Chen, Y. Hu, and B. Du, Suspension performance with one damper and one inerter, in Proceedings of the 24th Chinese Control and Decision Conference, Taiyuan, China (2012) 3534-3539.

[13] M. Z. Q. Chen, K. Wang, Z. Shu, C. Li, Realizations of a special class of admittances with strictly lower complexity than canonical forms, IEEE Trans. Circuits and Systems-I: Regular Papers 60 (2013) 2465-2473.

[14] M. Z. Q. Chen, K. Wang, Y. Zou, J. Lam, Realization of a special class of admittances with one damper and one inerter for mechanical control, IEEE Trans. Automat. Control 58 (2013) 1841-1846.

[15] M. Z. Q. Chen, K. Wang, M. Yin, C. Li, Z. Zuo, and G. Chen, Synthesis of n-port resistive networks containing 2n terminals, International Journal of Circuit Theory and Applications, in press (DOI: 10.1002/cta.1951).

[16] J. E. Colgate, Strictly positive real admittances for coupled stability, Journal of the Franklin Institute 329 (1992) 429-444.

[17] S. Evangelou, D. J. N. Limebeer, R. S. Sharp, M. C. Smith, Control of motorcycle steering instabilities-passive mechanical compensators incorporating inerters, IEEE Control Syst. Mag. 26 (2006) 78-88.

[18] R. M. Foster, Academic and theoretical aspects of circuit theory, in Proceedings of the IRE 50 (1962) 866-871.

[19] R. M. Foster, Biquadratic impedances realizable by a generalization of the five-element minimum-resistance bridges, IEEE Trans. Circuit Theory 10 (1963) 363-367.

[20] E. A. Guillemin, Synthesis of Passive Networks, John Wiley \& Sons, 1957.

[21] Y. Hu, C. Li, and M.Z.Q. Chen, Optimal control for semi-active suspension with inerter, in Proceedings of the 31st Chinese Control Conference, Hefei, China (2012) 2301-2306.

[22] J. Z. Jiang, Passive electrical and mechanical network synthesis, Ph.D. dissertation, University of Cambridge, Cambridge, UK, 2010.

[23] J. Z. Jiang, M. C. Smith, Regular positive-real functions and five-element network synthesis for electrical and mechanical networks, IEEE Trans. Automat. Control 56 (2011) 1275-1290. 
[24] J. Z. Jiang, M. C. Smith, Series-parallel six-element synthesis of biquadratic impedances, IEEE Trans. Circuits and Systems-I: Regular Papers 59 (2012) 2543-2554.

[25] J. Z. Jiang, A. Z. Matamoros-Sanchez, R. M. Goodall, M. C. Smith, Passive suspensions incorporating inerters for railway vehicles, Vehicle System Dynamics 50 (2012) 263-276.

[26] R. Kalman, Old and new directions of research in system theory, in Perspectives in Mathematical System Theory, Control, and Signal Processing, New York: Springer, 398 (2010) 3-13.

[27] H. K. Kim, Transformerless zero removal synthesis, Journal of the Franklin Institute 282 (1966) 242-259.

[28] Y. H. Ku, S. D. Bedrosian, On topological approaches to network theory, Journal of the Franklin Institute 279 (1965) 11-21.

[29] E. L. Ladenheim, A synthesis of biquadratic impedances, Master's thesis, Polytechnic Inst. of Brooklyn, N.Y., 1948.

[30] E. L. Ladenheim, A special biquadratic structure, IEEE Trans. Circuit Theory 10 (1963) 455-456.

[31] E. L. Ladenheim, Three-reactive five-element biquadratic structures, IEEE Trans. Circuit Theory 11 (1964) 88-97.

[32] P. M. Lin, A theorem on equivalent one-port networks, IEEE Trans. Circuit Theory 12 (1965) 619-621.

[33] H. J. Marquez, C. J. Damaren, Analysis and synthesis of strictly positive real transfer functions, Journal of the Franklin Institute 2 (1996) 245-256.

[34] J. G. Nickel, J. E. Schutt-Aine, Topological realizability conditions and their interpretation for admittance matrices of an MTL system with mode delays, Journal of the Franklin Institute 344 (2007) 912-917.

[35] C. Papageorgiou, M. C. Smith, Positive real synthesis using matrix inequalities for mechanical networks: application to vehicle suspension, IEEE Trans. Control Syst. Technol. 14 (2006) 423-435.

[36] F. M. Reza, Restrictions of the derivatives of positive real functions, Journal of the Franklin Institute 312 (1981) 335-343.

[37] H. C. Reddy, C. P. Reddy, M. N. S. Swamy, V. Ramachandran, Synthesis of RC transfer immittance functions with a minimum number of capacitors, Journal of the Franklin Institute 313 (1982) 197-206.

[38] S. Seshu, Minimal realization of the biquadratic minimum function, IRE Trans. Circuit Theory 6 (1959) 345350.

[39] S. Seshu, M. B. Reed, Linear Graphs and Electrical Networks, Addison-Wesley, 1961.

[40] M. C. Smith, Synthesis of mechanical networks: the inerter, IEEE Trans. Automat. Control 47 (2002) 16481662.

[41] M. C. Smith, F.-C. Wang, Performance benefits in passive vehicle suspensions employing inerters, Vehicle System Dynamics 42 (2004) 235-257.

[42] C. G. Vasiliu, Three-reactive five-element structures, IEEE Trans. Circuit Theory 16 (1969) 99.

[43] C. G. Vasiliu, Series-parallel six-element synthesis of the biquadratic impedances, IEEE Trans. Circuit Theory, 17 (1970) 115-121.

[44] C. G. Vasiliu, Four-reactive six-element biquadratic structures, IEEE Trans. Circuit Theory, 19 (1972) $530-531$.

[45] K. Wang, M. Z. Q. Chen, Realization of biquadratic impedances with at most four elements, in Proceedings of the 24th Chinese Control and Decision Conference, Taiyuan, China (2012) 2900-2905.

[46] K. Wang, M. Z. Q. Chen, Generalized series-parallel RLC synthesis without minimization for biquadratic impedances, IEEE Trans. Circuits and Systems II: Express Briefs 59 (2012) 766-770.

[47] F.-C. Wang, M.-K. Liao, The lateral stability of train suspension systems employing inerters, Vehicle System Dynamics 48 (2010) 619-643.

[48] F.-C. Wang, H. A. Chan, Vehicle suspensions with a mechatronic network strut, Vehicle System Dynamics 49 (2011) 811-830.

[49] F.-C. Wang, M.-R. Hsieh, H.-J. Chen, Stability and performance analysis of a full-train system with inerters, Vehicle System Dynamics 50 (2012) 545-571.

[50] Y.-S. Wu, D. P. Brown, New coefficient interrelations of RLC one-port driving functions, Journal of the Franklin Institute 282 (1966) 389-398.

[51] Q.-Z. Zha, W.-K. Chen, Transmission zeros and the two-port parameters, Journal of the Franklin Institute 334 (1997) 599-609. 